\title{
Faecal calprotectin levels in a high risk population for colorectal neoplasia
}

O Kronborg, M Ugstad, P Fuglerud, B Johne, J Hardcastle, J H Scholefield, K Vellacott, V Moshakis, J R Reynolds
The protein calprotectin (a $36.5 \mathrm{kD}$ nonglycosylated human protein) constitutes approximately $60 \%$ of the soluble cytoplasmic proteins in neutrophilic granulocytes and has been found in increased concentrations in faeces from symptomatic patients with colorectal cancer (CRC), inflammatory bowel disease, and certain infections. ${ }^{1}$ The increased concentrations in patients with CRC is probably not related to intestinal bleeding but may reflect release from surrounding leucocytes. ${ }^{2}$ Calprotectin in faeces seems to be a more sensitive marker for CRC than faecal occult blood ${ }^{3}$ but its specificity may be too low for screening average risk populations.

The sensitivity for detection of colorectal neoplasia may be increased by rehydration of the standard guaiac test Hemoccult-II, ${ }^{4}$ or by using a more sensitive faecal occult blood test such as the HemoccultSENSA ${ }^{5}{ }^{6}$ or the immunochemical test HemeSELECT. ${ }^{78}$ However, results have always been obtained at the cost of specificity.

The main purpose of the present study was to measure the sensitivity and specificity of calprotectin for detection of possible precursors of CRC (adenomas) in patients undergoing colonoscopy, the gold standard for comparison. Faecal occult blood tests are known to have a very low sensitivity for detection of adenomas, ${ }^{9}$ and the high sensitivity (87-91\%) of calprotectin for detection of $\mathrm{CRC}^{10}$ suggests that the same could be true for adenomas. Furthermore, we evaluated within patient changes in calprotectin concentrations in faeces from before to after removal of adenomas. In addition, between and within stool variability of concentrations of calprotectin was investigated. (cancer+adenoma). Specificity varied from $71 \%$ for one stool sample to $63 \%$ for four samples. Stool variability was small, suggesting that two spots from one stool were as discriminative as two spots from each of two stools.

Conclusions-The sensitivity and specificity of faecal calprotectin levels as a marker for colorectal adenoma and carcinoma justifies its use in high risk groups, but specificity is too low for screening of average risk persons. Lack of a decrease in levels after polypectomy may be due to a more widespread leucocyte migration into the intestinal lumen than that at the polyp site, and needs further investigation. (Gut 2000;46:795-800)

Keywords: calprotectin; colonoscopy; colorectal cancer; polyps; screening; tumour markers

\section{Methods}

The study was planned as an open multicentre trial with approximately 1000 patients from whom stool samples were collected before colonoscopy. Patients with adenomas also gave stool samples after polypectomy. Analyses of calprotectin were blinded and the endoscopist was unaware of the results.

The study comprised a high risk group for adenomas, reducing the number of patients necessary to obtain reasonable confidence limits for sensitivity and specificity. Inclusion criteria were at least one of the following: positive faecal occult blood test; participation in adenoma or carcinoma surveillance pro-

Abbreviations used in this paper: $\mathrm{CRC}$, colorectal cancer. 
gramme; polyp detected by sigmoidoscopy or barium enema; and participation in a family history screening programme.

Patients were at least 18 years of age and gave informed consent to take part in the study before colonoscopy. The study was approved by the local ethics committees. Exclusion criteria were: patients with respiratory tract infection; known inflammatory or neoplastic gastrointestinal disease, including ulcerative colitis, Crohn's disease, acute diverticulitis, colorectal cancer, and other malignant tumours of the small and large intestine; bacterial infection of the gut; and acute cholecystitis, cholangitis, and pancreatitis. The medical history included information on previous colorectal resection, rheumatic disease, intake of NSAIDs, anticoagulants, antibiotics and iron supplements, smoking, and alcohol consumption.

Five surgical hospital departments took part in the study and calprotectin analyses were performed in two departments of clinical chemistry and also at the Nycomed Allergen Laboratorium as a further check. A quality control programme of the latter was used to follow the laboratories of the clinical departments.

Two faecal samples from different parts of each of two stools were analysed. The samples were obtained on two consecutive days before bowel preparation and within seven days before colonoscopy. Patients were asked to deliver further samples 6-12 weeks after polypectomy when ulceration was believed to have disappeared.

Tubes were filled with $10-15 \mathrm{~g}$ of faeces and were sent to the laboratory within one day of sampling; patients were asked to keep samples refrigerated until delivery. Calprotectin is stable in faeces for three days at room temperature. At the laboratory samples were kept in a freezer $\left(-20^{\circ} \mathrm{C}\right)$ until analysis. Two samples of $5 \mathrm{~g}$ from each stool were tested using the PhiCal ELISA test device, produced by Nycomed Pharma AS. ${ }^{2}$ Essentially, $5 \mathrm{~g}$ of faeces were mixed with $10 \mathrm{ml}$ extraction solution (Tris buffered isotonic saline with 10 $\mathrm{mM} \mathrm{CaCl}, \mathrm{pH} 8.4$ ) in a high speed homogeniser, the homogenate was then centrifuged for 20 minutes $(10000 \mathrm{~g})$ before the supernatant was harvested, and calprotectin levels were measured using an ELISA assay. The lower limit of the analytical method was $3.75 \mathrm{mg} / 1$ and all observations with a value less than 3.75 were assigned a value of $3.75 \mathrm{mg} / \mathrm{l}$.

Polyps were measured (largest diameter) immediately after removal and classified according to the histopathological criteria described previously. ${ }^{11}{ }^{12}$ Evaluation of the sensitivity of calprotectin was based on the presence of adenomas and other subgroups (including patients with at least one adenoma with moderate to severe dysplasia). The latter group was expected to comprise 55 of 250 patients with adenomas - that is, a point estimate of the sensitivity in this subgroup of $90 \%$ would indicate a lower limit of $80 \%$ for the exact $95 \%$ confidence interval and a $95 \%$ probability of a true sensitivity of at least $80 .^{3}$
Table 1 Patients included in the analysis of sensitivity and specificity (sex distribution and age)

\begin{tabular}{|c|c|c|c|c|}
\hline & $\begin{array}{l}\text { Negative } \\
\text { colonoscopy }\end{array}$ & $\begin{array}{l}\text { Polyps } \\
\text { without } \\
\text { neoplasia }\end{array}$ & Adenomas & Carcinomas \\
\hline \multicolumn{5}{|c|}{ No of patients } \\
\hline Female & 231 & 39 & 71 & 12 \\
\hline Male & 257 & 61 & 132 & 11 \\
\hline Total & 488 & 100 & 203 & 23 \\
\hline \multicolumn{5}{|l|}{ Age (y) } \\
\hline Mean & 63.1 & 61.8 & 65.0 & 67.2 \\
\hline Range & $22-89$ & $34-84$ & $35-83$ & $46-84$ \\
\hline
\end{tabular}

The specificity of calprotectin was evaluated by using all patients without colorectal neoplasia and also all patients without polyps (adenomas and non-adenomas) or carcinomas. ROC analysis was used to illustrate the relation between sensitivity and specificity.

To evaluate the optimal sampling method for measuring calprotectin levels, a single stool sample and a maximum of several stool samples, obtained before colonoscopy, were examined.

Descriptive statistics included median values and $95 \%$ confidence intervals (CI) of the median.

\section{Results}

Initially 928 patients were included in the trial but 114 did not fulfil all of the inclusion criteria and hence 814 patients were included in the analysis. The main reason for exclusion was incomplete colonoscopy (101 patients). All 814 patients were recruited from January 1996 to April 1997 with varying numbers of patients from the five different centres $(537,73,32,71$, and 101).

Patients were allocated to four groups: those with no polyps or cancer; those with only nonneoplastic polyps; those with adenomas with or without non-neoplastic polyps; and those with carcinoma regardless of accompanying polyps (table 1).Three patients were Asian and the remainder were Caucasian.

Indications for colonoscopy were: positive faecal occult blood test (25 patients); surveillance for previous colorectal adenomas (605

Table 2 Characteristics of adenomas in 203 patients

\begin{tabular}{|c|c|c|}
\hline & No patients & No adenomas \\
\hline \multicolumn{3}{|l|}{ Multiplicity } \\
\hline 1 adenoma & 131 & \\
\hline 2 adenomas & 43 & \\
\hline$\geqslant 3$ adenomas & 29 & \\
\hline Total & 203 & \\
\hline \multicolumn{3}{|l|}{ Location } \\
\hline Caecum and ascending colon & & 67 \\
\hline Transverse colon & & 53 \\
\hline Descending colon & & 26 \\
\hline Sigmoid colon & & 111 \\
\hline $\operatorname{Rectum}(\leqslant 15 \mathrm{~cm})$ & & 68 \\
\hline Total & & 325 \\
\hline \multicolumn{3}{|l|}{ Size $(\mathrm{mm})$} \\
\hline$<10$ & & 234 \\
\hline $10-19$ & & 64 \\
\hline$\geqslant 20$ & & 29 \\
\hline Total & & 327 \\
\hline \multicolumn{3}{|l|}{ Worst histopathology } \\
\hline Tubular & 157 & \\
\hline Tubulovillous/villous & 46 & \\
\hline Total & 203 & \\
\hline \multicolumn{3}{|l|}{ Degree of dysplasia } \\
\hline Mild & & 217 \\
\hline Moderate & & 90 \\
\hline Severe & & 12 \\
\hline Total & & 319 \\
\hline
\end{tabular}




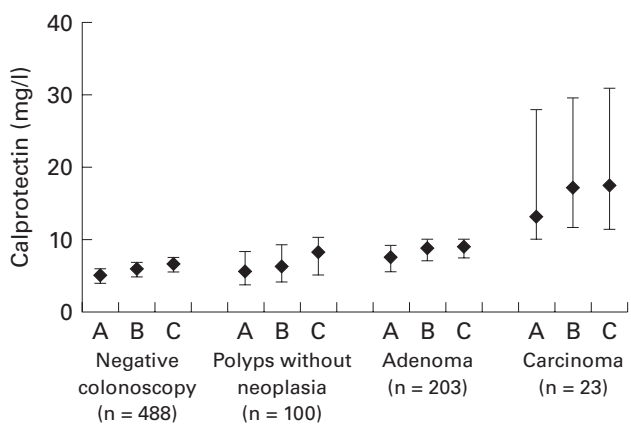

Figure 1 Median (95\% confidence intervals) faecal calprotectin levels in the four groups using the three sampling methods: $A$, one spot; $B$, maximum of two spots in the first stool; $C$, maximum of one spot from two stools.

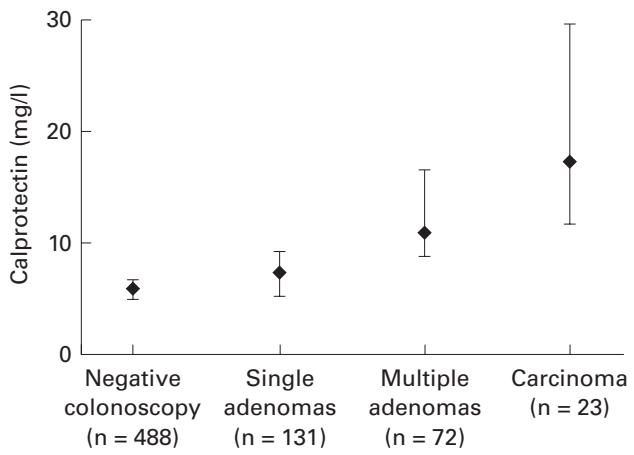

Figure 2 Faecal calprotectin levels by number of adenomas.

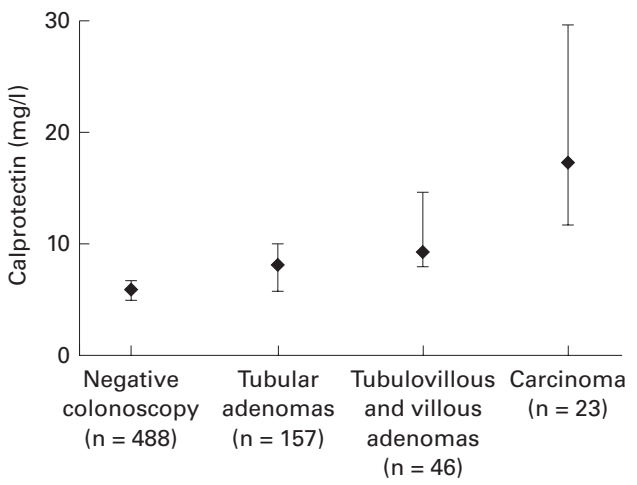

Figure 3 Faecal calprotectin levels by histology.

patients); polyp found by sigmoidoscopy or barium enema (130 patients); and family risk (54 patients). Colorectal resection had been performed previously in 202 patients. NSAIDs were used regularly by 102 patients. Patients with non-neoplastic polyps had one (73) or more (17) polyps. Patients with adenomas are described in table 2 ; the numbers are not identical in each of the subgroups because of missing data for a small number of adenomas.

Median calprotectin values were significantly higher in patients with cancer compared with the other three groups (fig 1) using the first spot of the first stool, a maximum of two spots in the first stool, or a maximum of the first spot in the first stool and the first spot in the second stool.

Patients with adenomas had significantly higher median calprotectin values than patients who had a negative result on colonoscopy when the two maximum values were used for comparison (fig 1). The difference tended to be significant when only the first spot of the first stool was used. However, values in patients with non-neoplastic polyps did not differ from those in patients with adenomas.

The 95\% CI for calprotectin values in patients who had a negative result on colonoscopy were less than those in patients with two or more adenomas and in patients with tubulovillous/villous adenomas using all three sampling methods (figs 2, 3). The same was true for the $95 \%$ CI for adenoma patients with a moderate/high degree of dysplasia but only using the maximum of the first spot of the first stool and the first spot of the second stool sampling methods. There were no significant differences between levels of calprotectin in different sized adenomas or in different locations.

The 95\% CI for calprotectin concentrations in patients who had a negative result on colonoscopy were less than those in patients with adenomas $\geqslant 10 \mathrm{~mm}$ with a moderate to high degree of dysplasia using the maximum of the first spot in the first stool and the first spot in the second stool sampling methods.

Variation in calprotectin levels in those who underwent colonoscopy was not related to daily smoking or alcohol intake, NSAIDs, or previous colorectal surgery. However, diverticulosis tended to increase the levels (7.75 $\mathrm{mg} / 1$ (95\% CI 5.67-9.63) v $4.98 \mathrm{mg} / 1$ (4.485.96)) but not more than the levels seen in patients with polyps without neoplasia (fig 1).

Specificity for patients who had a negative result on colonoscopy was assessed for the three sampling methods outlined in fig 1 and

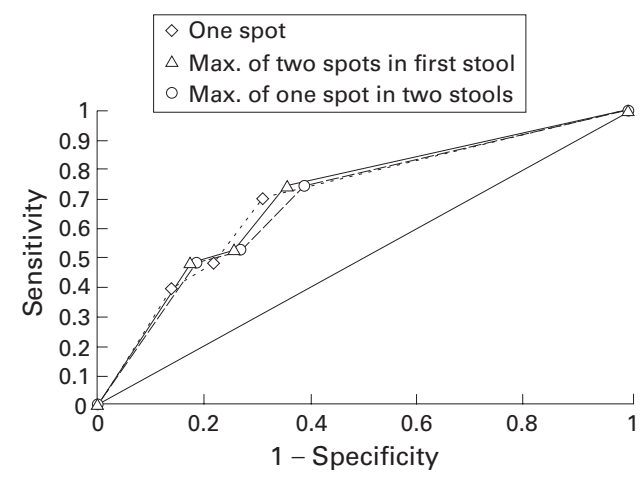

Figure 4 Sensitivity and specificity of the three sampling methods for asymptomatic colorectal cancer.

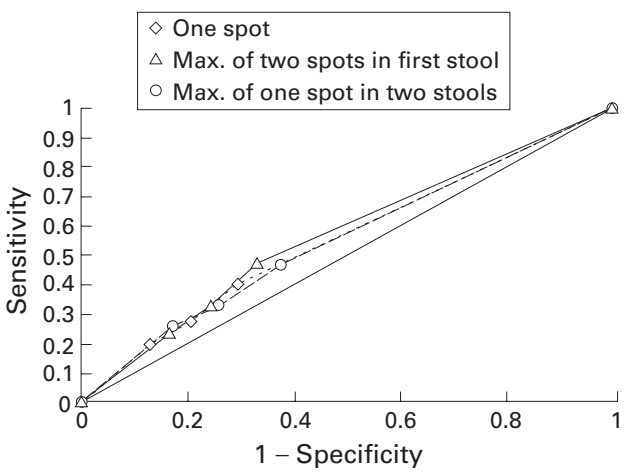

Figure 5 Sensitivity and specificity of the three sampling methods for asymptomatic colorectal neoplasia. 
Table 3 Sensitivity (\%) of faecal calprotectin with a cut off value of $10 \mathrm{mg} / \mathrm{l}$ in subgroups of patients with adenomas

\begin{tabular}{llll}
\hline & \multicolumn{2}{l}{ Sampling method } \\
\cline { 2 - 4 } & First spot & $\begin{array}{l}\text { Max of two spots } \\
\text { from first stool }\end{array}$ & $\begin{array}{l}\text { Max of one spot from } \\
\text { each of two stools }\end{array}$ \\
\hline Single adenoma $(\mathrm{n}=131)$ & 30 & 37 & 37 \\
Multiple adenomas $(\mathrm{n}=72)$ & 50 & 56 & 54 \\
Largest adenoma $<10 \mathrm{~mm}(\mathrm{n}=131)$ & 37 & 45 & 46 \\
Largest adenoma $>20 \mathrm{~mm}(\mathrm{n}=25)$ & 48 & 48 & 56 \\
Right colon $(\mathrm{n}=46)$ & 30 & 37 & 42 \\
Left colon $(\mathrm{n}=130)$ & 35 & 43 & 63 \\
Both $(\mathrm{n}=27)$ & 57 & 59 & \\
\hline
\end{tabular}

Table 4 Calprotectin levels before and after adenoma resection *

\begin{tabular}{llrrrr}
\hline & \multicolumn{4}{c}{ After polypectomy } \\
\cline { 3 - 6 } & $\begin{array}{l}\text { Calprotectin level } \\
\text { intervals }\end{array}$ & $<10 \mathrm{mg} / \mathrm{l}$ & $\geqslant 10$ and $<20$ & $\geqslant 20 \mathrm{mg} / \mathrm{l}$ & Total \\
\hline \multirow{2}{*}{ Before polypectomy } & $<10 \mathrm{mg} / \mathrm{l}$ & 77 & 9 & 8 & 94 \\
& $\geqslant 10 \mathrm{and}<20$ & 10 & 9 & 7 & 26 \\
& $\geqslant 20 \mathrm{mg} / \mathrm{l}$ & 6 & 6 & 14 & 26 \\
& Total & 93 & 24 & 29 & 146 \\
\hline
\end{tabular}

*Mean faecal calprotectin values before and 6-12 weeks after polypectomy were calculated for each patient. The table shows number of patients in each calprotectin interval.

calculated as 100 times the number of observations less than or equal to the limit (three different cut off points: 10, 15, and 20 $\mathrm{mg} / \mathrm{l}$ ) divided by the total number of observations in the group (figs 4,5). For all cut off points, specificity was highest when the first spot of the first stool was used. Including patients with non-adenomatous polyps resulted in nearly the same specificity, deviating by $1-2 \%$.

The sensitivity for detection of adenomas and cancer was calculated as 100 times the number of observations greater than the cut off point divided by the total number of observations in the group (figs 4,5 ). Sensitivity was always higher for cancer than for adenomas and was lowest when one spot was used from the first stool. The second spot from the second stool did not improve the diagnostic value (results not shown but were used for analysis of spot variation).

Grouping patients according to the different characteristics of the adenomas did not influence the sensitivity for the degree of dysplasia or histological structure; however, sensitivity increased with multiple adenomas, increase in size, and number of locations (table 3 ).

One hundred and forty six adenoma patients delivered stools both before and after polypectomy. There was no significant change in calprotectin levels for all adenoma patients or for the subgroups (before: median 6.82 (95\% CI 6.03-8.21; after: median 7.07 (5.26-8.67)) (table 4).

\section{Discussion}

Many colonoscopies have been performed in the past two decades as part of surveillance programmes in high risk groups. The benefit of colonoscopy has been demonstrated by the reduction in mortality from CRC. ${ }^{13}$ However, the use of endoscopic resources has been substantial and simpler and less expensive surveillance markers such as Hemoccult-II have been examined. However, the latter was found to have a sensitivity for new CRC of only 2/11 $(18 \%)$ and of $31 / 186(16 \%)$ for adenomas when used as a surveillance technique after previous colorectal neoplasia in 1572 colonoscopies. ${ }^{9}$ Blood loss from adenomas is small, especially from those less than $10 \mathrm{~mm}$, explaining the low sensitivity. Sensitivity reached $40 \%$ in patients with adenomas $\geqslant 20$ $\mathrm{mm}$ in diameter and $26 \%$ in patients with $\geqslant 2$ adenomas. Our data suggest that calprotectin might be a more sensitive marker $(69-73 \%$ for CRC and $36-43 \%$ for adenomas using $10 \mathrm{mg} / 1$ as the cut off value). ${ }^{9}$ However, this is clearly at the cost of specificity, which was much lower in our 488 patients who had a negative result on colonoscopy compared with the 1094 patients in the Hemoccult-II study. ${ }^{9}$ Rehydration of the Hemoccult-II test has resulted in detection of a higher number of carcinomas and adenomas in asymptomatic patients, ${ }^{4}$ suggesting a higher sensitivity than the conventional Hemoccult-II. Again, this was at the expense of a decrease in specificity as demonstrated by an increase in false positive tests. ${ }^{4}$

A more sensitive guaiac test, the HemoccultSENSA, demonstrated sensitivity for CRC and adenomas $\geqslant 10 \mathrm{~mm}$ of $94 \%$ ad $60 \%$, respectively, ${ }^{5}$ which appear to be better than the results obtained with calprotectin. However, the cancer patients were symptomatic. Patients scheduled for health appraisal at the Kaiser Permanente Medical Center ${ }^{6}$ demonstrated a sensitivity for CRC of $79 \%$ and for adenomas $\geqslant 1 \mathrm{~cm}$ of $68 \%$. However, this population may not represent an asymptomatic screening population. Similar or better results than those for calprotectin have been obtained using the immunochemical test Heme SELECT but with positivity rates of 3.7$13.0 \%$ in large studies (1304-12 699 patients) suggesting low specificity. ${ }^{7}$

A recent study ${ }^{14}$ involving a similarly high risk group as the present study demonstrated a positivity rate of $20 \%$ for HemeSELECT in 808 individuals; sensitivity for CRC and adenomas $\geqslant 1 \mathrm{~cm}$ was $70 \%$ and $44 \%$, respectively, values similar to those obtained for calprotectin. A subset of 417 of the 808 persons also completed Hemoccult-II tests with sensitivities of $33 \%$ and $18 \%$. The specificity for HemeSELECT was somewhat higher $(88 \%)$ than that for calprotectin $(63-71 \%)$ but lower than that for Hemoccult (98\%). Nevertheless, it was concluded that Heme SELECT was a more satisfactory surveillance tool than Hemoccult-II in high risk groups. A population study with HemeSELECT confirmed a sensitivity of $69 \%$ for CRC and almost the same for adenomas $\geqslant 1 \mathrm{~cm}(66 \%)^{6}{ }^{8}$; specificity was not less than $95 \%$. An Australian study ${ }^{5}$ involving 81 patients with predominantly asymptomatic adenomas demonstrated a sensitivity for adenomas $\geqslant 10 \mathrm{~mm}$ of $76 \%$. The HemeSELECT test has been automated in Japan making it an attractive test for high risk groups; the same may be possible for calprotectin.

A simpler immunochemical test, FlexSure OBT, has been developed, needing only office procedures, but this test cannot be automated. ${ }^{8}$ 
The same problem exists with the combined test, when a positive HemoccultSENSA is followed by a HemeSELECT test. The advantage of the latter would be increased specificity compared with the HemoccultSENSA alone but the sensitivity for CRC was less $(65 \%)^{6}$ than that in the present study for calprotectin ( $71 \%)$; the sensitivity for adenomas $\geqslant 10 \mathrm{~mm}$ was similar (50\%).

Dietary restrictions are not required for measurement of calprotectin levels, which is an advantage over the Hemoccult-II and HemoccultSENSA. The readability of the calprotectin test results ${ }^{8}$ is not a problem. However, a major drawback of the calprotectin test is the sampling procedure which is more inconvenient to patients than the test cards of faecal occult blood tests. Recently, this problem has been addressed by the use of a small double tube, reducing the stool sample to $50-100 \mathrm{mg} .{ }^{15}$ Refrigeration is unnecessary if samples are brought to the laboratory within three days. Respiratory tract infection may or may not affect faecal calprotectin levels. No systematic study has been performed to rule out this possibility.

Our sampling methods for measurement of faecal calprotectin levels suggest that two spots from one stool sample is nearly as discriminate as repeating the procedure to obtain four spots from two stools. This was not unexpected from previous analyses showing a small between stool coefficient of variation $(22 \%)$ compared with that for haemoglobin $(80 \%)$ in patients with symptomatic colorectal cancer. ${ }^{16}$ However, a recent study has suggested that high risk groups for colorectal cancer may include subgroups with marked day to day variability and no neoplasia or chronic inflammation. ${ }^{17}$ Even one spot did not reduce the sensitivity for detection of CRC by more than a few percentages, but two spots may be preferable to detect more adenomas. Cut off limits $>10 \mathrm{mg} / 1$ were clearly preferable to $20 \mathrm{mg} / \mathrm{l}$, resulting in a much higher sensitivity, although specificity decreased accordingly.

Surprisingly, there was no significant decrease in calprotectin levels after polypectomy despite postponing sampling for 6-12 weeks when inflammatory reactions would have disappeared. This finding suggests that the presence of granulocytes in faeces may not be related to the mass of adenomatous tissue but may depend more on a general intestinal mucosal defect, which also could explain the slightly elevated levels in patients with nonneoplastic polyps. This suggestion is supported by the finding of similar or even higher levels of calprotectin in patients who had undergone colonic resection for cancer. ${ }^{18}$ Migration of leucocytes into the lumen may be even higher in patients who have had cancer in spite of reduced intestinal volume following resection.

Further investigations are needed to clarify these findings; a recent study has demonstrated substantial colonisation by invasive $E$ coli strains in the colonic mucosa of patients with colorectal cancer as well as adenomas, but not in control subjects. ${ }^{19}$ The slightly elevated levels of calprotectin in patients with diverticu- losis may be explained by diverticulitis in some of these despite attempts to exclude such patients.

Faecal calprotectin and faecal occult blood are not specific markers of colorectal neoplasia, the former being a marker of inflammation and the latter a marker of bleeding. More specific molecular markers ${ }^{20}$ will probably not be available for general clinical use for several years; a panel of biomarkers is needed which identify neoplasia in the intestinal mucosa and can be detected in exfoliated colonocytes. There is some support for the use of CD $44,{ }^{21} \mathrm{~K}$-ras mutations, and mutations in the p53 or APC gene, ${ }^{22}$ but sensitivity values need to be increased before molecular methods can be used for screening. Colonoscopy and even sigmoidoscopy are expensive examinations which should be used as a first priority in symptomatic patients. Endoscopy may also carry a high priority as the first examination in some high risk groups such as HNPCC families and in those with ulcerative colitis.

In other high risk groups less invasive and less expensive procedures may be preferred for the initial examination. The non-specific faecal marker calprotectin may compete with the more sensitive faecal occult blood tests and automation will make it as attractive as HemeSELECT. However, both of these tests should only be considered in high risk groups such as surveillance after previous CRC or colorectal adenoma, and in families with one or two members with CRC or adenoma. Specificity is too low for screening of average risk populations.

We thank Ms Margrethe Goksøyr, Nycomed Pharma AS, Oslo, Norway, for skilful supervision and monitoring of laboratories and trial centres. Ms Kate Bostock, University Hospital, and trial centres. Ms Kate Bostock, University Hospital, Nottingham UK, and Ms Inger Lise Andersen, Odense Univer-
sity Hospital, are gratefully acknowledged for performance of sity Hospital, are gratefully acknowledged for performance of
the calprotectin analyses.

1 Johne B, Fagerhol MK, Lyberg T, et al. Functional and clinical aspects of the myelomonocyte protein calprotectin Mol Pathol 1997;50:113-23.

2 Røseth AG, Fagerhol MK, Aadland E, et al. Assessment of the neutrophil dominating protein calprotectin in feces. A methodologic study. Scand F Gastroenterol 1992;27:793-8.

3 Røseth AG, Kristinsson J, Fagerhol MK, et al. Faecal calprotectin: A novel test for the diagnosis of colorectal cancer? Scand f Gastroenterol 1993;28:1073-6.

4 Kewenter J, Björk S, Haglind E, et al. Screening and rescreening for colorectal cancer. A controlled trial of faecal occult blood testing in 27,700 subjects. Cancer 1988; 62:645-51.

5 St.John DJB, Young GP, Alexeyeff MA, et al. Evaluation of new occult blood tests for detection of colorectal neoplasia.

6 Allison JE, Tekawa IS, Ransom LJ, et al. A comparison of faecal occult-blood tests for colorectal cancer screening. $N$ Engl f Med 1996;334:155-9.

7 Robinson MHE, Marks CG, Farrands PA, et al. Screening for colorectal cancer with an immunological faecal occult blood test: 2 year follow-up. Br F Surg 1996;83:500-1.

8 Allison JE. Review article: Faecal occult blood testing for colorectal cancer. Aliment Pharmacol Ther 1998;12:1-10.

9 Jahn H, Jørgensen OD, Kronborg O, et al. Can HemoccultII $^{\mathrm{TM}}$ replace colonoscopy in surveillance after radical surgery for colorectal cancer and after polypectomy? Dis Colon Rectum 1992;35:253-6.

10 Kronborg O, Kristensson J, Tøn HI, Fuglerud P, Johne B. Fecal calprotectin levels in colorectal cancer patients and high risk subjects; a comparison with a new

11 O'Brien MJ, O'Keane JC, Zauber A, et al. Precursors of colorectal carcinoma. Biopsy and biologic markers. Cancer 1992;70:1317-27. 
12 Jørgensen $\mathrm{OD}$, Kronborg $\mathrm{O}$, Fenger C. The Funen adenoma follow-up study. Characteristics of patients and initial adenomas in relation to severe dysplasia. Scand $f$ Gastroenterol 1993;28:239-43.

13 Jørgensen OD, Kronborg O, Fenger C. The Funen adenoma follow-up study. Incidence and death of colorectal carcinoma in an adenoma surveillance program. Scand $\mathcal{F}$ Gastroenterol 1993;28:869-74

14 Robinson MHE, Kronborg O, Williams CB, et al. Faecal occult blood testing and colonoscopy in the surveillance of subjects at high risk of colorectal neoplasia. Br F Surg 1995; 82:318-20.

15 Kristinsson J, Nygaard K, Armbruster C, et al. Faecal calprotectin levels in colorectal cancer, measured with a new simplified method. Abstract World Congresses of Gastroenterology Vienna. Digestion 1998;59:730.

16 Gilbert JA, Ahlquist DA, Mahoney DW, et al. Faecal marker variability in colorectal cancer: calprotectin versus hemovariability in colorectal cancer: calprotectin ver.

17 Husebye E, Tøn H. Biological variation in faecal calprotectin concentration in patients submitted for colonoscopy. Abstract World Congresses of Gastroenterology Vienna. Digestion 1998;59:731.
18 Kristinsson J, Roseth A, Fagerhol MK, et al. Fecal calprotectin concentration in patients with colorectal carcinoma. Dis Colon Rectum 1998;41:316-21.

19 Swidsinski A, Khilkin M, Kerjaschki D, Schreiber S, Ortner $\mathrm{M}$, Weber J, Lochs H. Association between intraepithelial Escherichia coli and colorectal cancer. Gastroenterology 1998;115:281-6.

20 Dutta SK. Noninvasive detection of colorectal cancer by molecular tools: coming of age. Editorials Gastroenterol 1998;114:1333-4.

21 Yamao T, Matsumura Y, Shimada Y, et al. Abnormal expression of CD44 variants in the exfoliated cells in the feces of patients with colorectal cancer. Gastroenterology 1998;114:1196-205.

22 Nollau P, Wagener C. Detection of mutant alleles of tumour cells in stool: diagnostic value and perspectives of highly sensitive molecular methods for the early detection of colorectal cancer. In: Schmiegel W, Schölmerich J, eds. Colorectal cancer. Molecular mechanisms, premalignant state and its prevention. FALK Symposium vol 109. Dordrecht: Kluwer Academic Publishers, 1998:135-43. 